TRANSACTIONS OF THE

AMERICAN MATHEMA TICAL SOCIETY

Volume 180, June 1973

\title{
MAXIMAL REGULAR RIGHT IDEAL SPACE OF A PRIMITIVE RING. II
}

\author{
BY \\ KWANGIL KOH AND JIANG LUH
}

\begin{abstract}
If $R$ is a ring, let $X(R)$ be the set of maximal regular right ideals of $R$. For each nonempty subset $E$ of $R$, define the hull of $E$ to be the set $\{I \in X(R) \mid E \subseteq I\}$ and the support of $E$ to be the complement of the hull of $E$. Topologize $X(R)$ by taking the supports of right ideals of $R$ as a subbase. If $R$ is a right primitive ring, then $X(R)$ is homeomorphic to an open subset of a compact space $X\left(R^{\#}\right)$ of a right primitive ring $R^{\#}$, and $X(R)$ is a discrete space if and only if $X(R)$ is a compact Hausdorff space if and only if either $R$ is a finite ring or a division ring. Call a closed subset $F$ of $X(R)$ a line if $F$ is the hull of $I \cap J$ for some two distinct elements $I$ and $J$ in $X(R)$. If $R$ is a semisimple ring, then every line contains an infinite number of points if and only if either $R$ is a division ring or $R$ is a dense ring of linear transformations of a vector space of dimension two or more over an infinite division ring such that every pair of simple (right) $R$-modules are isomorphic.
\end{abstract}

Introduction. Let $R$ be a ring. A right ideal $I \subseteq R$ is called regular if there exists an $e \in R$ such that, for all $a \in R, a-e a \in I$. Let $X(R)$ be the set of maximal regular right ideals of $R$. If $E$ is a nonempty subset of $R$, the set of maximal regular right ideals of $R$ which contain $E$ is called the bull of $E$ and the complement of the hull of $E$ with respect to $X(R)$ is called the support of $E$. We topologize $X(R)$ by taking the set of supports of right ideals of $R$ as a subbase. Then the topological space $X(R)$ is called the maximal regular right ideal space of $R$ [3]. If $1 \in R, X(R)$ is a compact space [3, 1.7]; however, in general, it is not a compact space. Recall that a topological space $X$ is irreducible [4] if $X \neq \varnothing$ and $X$ is not the union of two proper closed subsets. $X$ is reducible if it is not irreducible. In [3], we have shown that if $R$ is a (right) primitive ring, then $X(R)$ is reducible if and only if $R$ is a dense ring with nonzero socle of linear transformations of a vector space of dimension two or more over a finite field, and $X(R)$ is a Hausdorff space if and only if either $R$ is a division ring or $X(R)$ is reducible and $R$ modulo its socle is a radical ring. In this paper we continue our previous work [3]. Our main results are as follows:

Received by the editors May 2, 1972.

AMS (MOS) subject classifications (1970). Primary 16A20, 16A42; Secondary 16A48.

Key words and phrases. Maximal regular right ideals, socle, irreducible spaces, compact Hausdorff spaces, lines, hyperplanes, support.

Copyright $\odot 1973$, American Mathematical Society 
If $R$ is a ring and $S$ is an ideal of $R$ then $X(S)$ is homeomorphic to an open subset of $X(R)$. If $R$ is a primitive ring then $X(R)$ is embeddable as an open subset of a compact space $X\left(R^{\#}\right)$ for some primitive ring $R^{\#}$. As a consequence of this fact, we will be able to show that if $R$ is a primitive ring then $X(R)$ is a compact Hausdorff space if and only if $X(R)$ is a discrete space if and only if either $R$ is a division ring or a finite ring. For any subset $Y$ of $X(R)$, define $j(y)=$ $\bigcap\{I \in X(R) \mid I \in Y\}$.

Let us call a closed subset $F$ of $X(R)$ a line if $F$ is the hull of $j(\{x\}) \cap j(\{y\})$ for some distinct points $x, y$. Call a closed subset $F$ of $X(R)$ a byperplane provided that $j(F) \neq J(R)$, the Jacobson radical, and if $F^{\prime}$ is a closed subset such that $F^{\prime} \supseteq F$ and $F^{\prime} \neq F$ then $j\left(F^{\prime}\right)=J(R)$. If $R$ is a primitive ring, then $R$ has nonzero socle if and only if $X(R)$ has a byperplane, and $R$ is simple artinian if and only if (i) $X(R)$ is compact, (ii) $X(R)$ has no line which contains exactly two points, and (iii) $X(R)$ has a hyperplane. For a semisimple ring $R, X(R)$ contains at least two points and every line of $X(R)$ contains an infinite number of points if and only if $R$ is a dense ring of linear transformations of a vector space of dimension two or more over an infinite division ring such that every pair of simple (right) $R$-modules are isomorphic. Throughout this paper, by a primitive ring we always mean a right primitive ring and every module is a right module.

1. Preliminaries.

1.1. Definition. If $E$ is a subset of $R$, we define the support of $E$ to be the set of maximal regular right ideals of $R$ which do not contain the set $E$. It will be denoted by $\operatorname{supp}(E)$.

1.2. Definition. If $R$ is a ring, let $X(R)$ be the set of maximal regular right ideals of $R$. We give a topology to $X(R)$ which is generated by the subbasis consisting of all supports of the right ideals of $R$. We will call $X(R)$ together with this topology the maximal regular right ideal space of $R$. It will simply be denoted by $X(R)$.

1.3. Definition. If $E$ is a subset of $R$, the bull of $E$ is the set $X(R) \backslash \operatorname{supp}(E)$. It will be denoted by $b(E)$. If $E=\{e\}$ is a singleton set, we let $b(e)=b(E)$.

1.4. Definition. A nonempty topological space $X$ is irreducible if $X$ is not the union of two proper closed subsets. $X$ is reducible if it is not irreducible. A subset $Y$ of a space $X$ is irreducible if $Y$ is irreducible in the induced topology.

1.5. Definition. If $x$ is an element of $X(R)$ for some ring $R$, then $x$ is also a right ideal of $R$. To make a distinction, we write $j(x)$ for the right ideal $x$. If $Y$ is a subset of $X(R)$, we define $j(Y)=\bigcap\{j(x) \mid x \in Y\}$. If $Y=\{y\}$ a singleton set, we let $j(y)=j(Y)$.

1.6. Definition. Let $R$ be a ring and let $V$ be a right $R-$ module. For each $v \in V$, we define $v^{\perp}=\{r \in R \mid v r=0\}$. If $E$ is a subset of $V$, we define $E^{\perp}=$ $\bigcap\left\{v^{\perp} \mid v \in E\right\}$. 
1.7. Proposition. Let $R$ be a ring and I be a maximal regular right ideal of $R$. If $A, B$ are right ideals of $R$ such that $A B \subseteq I$, then either $A \subseteq I$ or $B \subseteq I$.

Proof. Suppose that $A \not \subset I$ and $B \not \subset I$. Then $A+I=R$ and $R B=(A+I) B$ $\subseteq I$. Let $e$ be a left identity modulo $I$. Since $e b \in I$, for every $b \in B, B \subseteq I$. This is a contradiction.

1.8. Corollary. Let $R$ be a ring and $I$ be a maximal regular right ideal of $R$. If $a \in R$ such that $a R \subseteq I$, then $a \in I$.

Proof. Let $A=\{a \in R \mid a R \subseteq I\}$. Then $A$ is a right ideal of $R$ and $A R \subseteq I$. Hence by $1.7, A \subseteq I$ and $a \in I$.

1.9. Corollary. Let $R$ be a ring and $S$ be an ideal of $R$. If $m$ is a maximal regular right ideal of $S$ then $m$ is a right ideal of $R$.

Proof. Let $a$ be an element of $m$ and let $r$ be an element of $R$. Then $(a r) S$ $=a(r S) \subseteq m$. Hence by 1.8 , ar $\in m$.

1.10. Proposition. Let $S$ be an ideal of a ring $R$. Let $m$ be a maximal regular right ideal of $S$ and let $f$ be a left identity modulo $m$ in $S$. Define $I(m, f)=$ $\{r \in R \mid f r \in m\}$. Then $I(m, f)$ is a maximal regular right ideal of $R$ such that $I(m, f) \cap S=m$. If $e$ is another left identity modulo $m$ in $S$, then $I(m, e)=$ $I(m, f)$.

Proof. By 1.9, $m$ is a right ideal of $R$. Hence $I(m, f)$ is a right ideal of $R$. Since $f(f r-r)=f(f r)-f r \in m$ for every $r \in R, f$ is a left identity modulo $I(m, f)$. Clearly $m \subseteq I(m, f)$. Since $f s-s \in m$ for every $s \in S, m=I(m, f) \cap S$. If $I(m, f)$ were not maximal, then there would exist a maximal right ideal, say $M$ of $R$, such that $I(m, f) \subseteq M$ but $I(m, f) \neq M$. Let $a \in M$ such that $a \notin I(m, f)$. Then $f a \notin m$ and $f a \in M$. Hence $m \subseteq M \cap S$ but $m \neq M \cap S$. Since $M \cap S$ is a right ideal of $S$ and $m$ is maximal, $M \cap S=S$ and $f \in M$. Thus $R=M$. This is a contradiction. In order to prove that $I(m, f)=I(m, e)$, we observe that $f s-s \in m$ and $s-e s \epsilon$ $m$ for every $s \in S$. This implies that $f s-e s=(f-e)_{s} \in m$ for every $s \in S$. Hence, by 1.8, $f-e \in m$ and $f r \in m$ if and only if $e r \in m$ for every $r \in R$.

1.11. Proposition. Let $R$ be a ring and $S$ be an ideal of $R$. If $I_{1}, I_{2}$ are maximal regular right ideals of $R$ sucb that $S \not I_{i}, i=1,2$, then $S \cap I_{i}$ is a maximal regular right ideal of $S$ for each $i, i=1,2$, and if $S \cap I_{1}=S \cap I_{2}$, then $I_{1}=I_{2}$.

Proof. Let $e_{i}$ be a left identity modulo $I_{i}$ for each $i$ where $i=1,2$. Since $S \not \subset I_{i}, S+I_{i}=R$ and $e_{i}=s_{i}+a_{i}$ for some $s_{i} \in S$ and $a_{i} \in I_{i}$. Then $s_{i}$ is a left 
identity modulo $I_{i} \cap S$ in $S$. Let $m$ be a maximal regular right ideal of $S$ such that $I_{i} \cap S \subseteq m$. Clearly, $s_{i}$ is a left identity modulo $m$. Hence by $1.10, m=$ $I\left(m, s_{i}\right) \cap S$ and $I_{i} \cap S \subseteq I \bar{l}\left(m, s_{i}\right) \cap S$. Since $S$ is a two-sided ideal of $R, I_{i} S$ $\subseteq I_{i} \cap S \subseteq I\left(m, s_{i}\right)$. Since $S \mathscr{E} I\left(m, s_{i}\right), I_{i} \subseteq I\left(m, s_{i}\right)$ by 1.7 and hence $I_{i}=$ $I\left(m, s_{i}\right)$. Thus $I_{i} \cap S=m$ and if $S \cap I_{1}=S \cap I_{2}$ then, by $1.10, I_{1}=I_{2}$.

1.12. Theorem. Let $R$ be a ring and $S$ be an ideal of $R$. Then $\operatorname{supp}(S)$ is bomeomorpbic to $X(S)$.

Proof. Define a function $\phi$ from supp $(S)$ into $X(S)$ by $\phi(I)=I \cap S$. Then $\phi$ is a bijection by 1.10 and 1.11 . Let $A$ be a right ideal of $S$. Then $\phi^{-1}(\operatorname{supp}(A))=\{I \in \operatorname{supp}(S) \mid A \not \subset I \cap S\}$. But this set is equal to $\operatorname{supp}(A R)$ in $X(R)$ which is contained in supp $(S)$. Thus $\phi$ is a continuous mapping. Since $S$ is a two-sided ideal, if $B$ is a right ideal of $R$ then $\operatorname{supp}(S) \cap \operatorname{supp}(B)=$ $\operatorname{supp}(B S)($ refer 1.7$)$ and $\phi(\operatorname{supp}(B S))=\{I \cap S \mid I \in X(R)$ and $B S \not \subset I\}$. This is clearly an open set in $X(S)$ and $\phi$ is an open mapping.

1.13. Proposition. If $R$ is a primitive ring, then there is a primitive ring $R^{*}$ with 1 such that $R$ is an ideal of $R^{*}$ and $X(R)$ is bomeomorphic to an open subset of $X\left(R^{*}\right)$.

Proof. Let $E=\mathbf{Z} \times R$, the usual extension ring of $R$, Let $I$ be a maximal right ideal of $E$ such that $R \notin I I$ and $R /(I \cap R)$ is a faithful simple $R$-module. If $E / I$ were not faithful, let $S$ be the largest ideal of $E$ which is contained in $I$. Then $S \cap R=0$ since $R /(I \cap R)$ is faithful. Let $R^{*}=E / S$. Then $R^{*}$ is a primitive ring with 1 and $R$ is isomorphic to an ideal of $R^{*}$. Hence by 1.12 , $X(R)$ is homeomorphic to an open subset of $X\left(R^{*}\right)$.

1.14. Definition. A ring $R$ is called (von Neumann) regular if and only if for every element $a$ in $R$ there is $b$ in $R$ such that $a b a=a$ (refer [6]).

1.15. Remark. If $R$ is (von Neumann) regular, then $a \in a R$ for every $a \in R$ even though $R$ may not contain a unit element and $a R=e R$ for some idempotent $e \in R$. von Neumann proved that if $R$ is a regular ring with 1 , then for any idempotent $e \in R$ and an element $b \in R, e R+(1-e) b R=g R$ for some idempotent $g \in R$ (refer [6]). Since $e R+(1-e) b R=e R+b R$, this means that any finitely generated right ideal of $R$ is principal. With a slight modification of von Neumann's proof for the above assertion, one can also conclude that if $R$ is a regular ring (not necessarily with 1) then for any idempotent $e \in R$ and an element $b \in R, e R+b R=e R+\{b r-e b r \mid r \in R\}=g R$ for some idempotent $g$ in $R$.

1.16. Theorem. If $R$ is a regular ring, then $X(R)$ is compact if and only if $1 \in R$. 
Proof. If $1 \in R$ then $X(R)$ is compact for every ring $R$ by $[3,1.7]$. Assume that $R$ is a regular ring and $X(R)$ is a compact space. Let $E$ be the set of idempotents in $R$. Then $X(R)=\bigcup_{e_{\alpha} \epsilon E} \operatorname{supp}\left(e_{a} R\right)$. Since $X(R)$ is compact, there exist $e_{a_{1}}, e_{a_{2}}, \cdots, e_{a_{n}}$, a finite number of idempotents in $R$, such that $X(R)=$ $\bigcup_{i=1}^{n} \operatorname{supp}\left(e_{a_{i}} R\right)$. Hence $\varnothing=\bigcap_{i=1}^{n} b\left(e_{a_{i}} R\right)=b\left(\sum_{i=1}^{n} e_{a_{i}} R\right)$. Let $e$ be an idempotent such that $\sum_{i=1}^{n} e_{a_{i}} R=e R$. If $e R \neq R$, then the right ideal $\{r-e r \mid r \in R\}$ is not zero. Let $b \in R$ be such that $b-e b \neq 0$. Then by 1.15 , there is an idempotent $g \in R$ such that $g R=e R+(b-e b) R$. Hence $g e=e$ and $g-e \neq 0$. Since $R$ is a semisimple ring, there is a simple right $R$-module $M$ such that $M(g-e) \neq 0$. Let $m \in M(g-e)$ such that $m \neq 0$. Then $m e R=0$ and $e R \subseteq m^{\perp}$, which is maximal regular right ideal. This means that $b(e R) \neq \varnothing$, which is a contradiction. Thus $e R=R$ and $e x=x$ for every $x \in R$. Since $(x-x e) R=0$ for every $x \in R, e=1$.

1.17. Theorem. If $R$ is a primitive ring, then $X(R)$ is a compact Hausdorff space if and only if $R$ is either a division ring or a finite ring.

Proof. If $R$ is either a division ring or a finite ring, then certainly $X(R)$ is a compact Hausdorff space. Suppose that $X(R)$ is a compact Hausdorff space. By $[3,2.5]$, if $S$ is the socle of $R$, then $R / S$ is a radical ring. Since $X(S)$ is homeomorphic to $\operatorname{supp}(S)$ and $X(R)=\operatorname{supp}(S), X(S)$ is a Hausdorff compact space. Since $S$ is a regular ring, by $1.16,1 \in S$ and $R=S$. Thus by [3, 2.7] either $R$ is a division ring or a finite ring.

2. Irreducible closed sets. If $R$ is a dense ring of linear transformations of a vector space, then an element $a \in R$ generates a minimal right ideal if and only if the rank of $a$ is 1 (refer $[1$, p. 76]). It is interesting to note that the following assertion is also true:

\subsection{Proposition. If $R$ is a dense ring of linear transformations of a left} vector space $V$ over a division ring $D$ and $A$ is a nonzero right ideal of $R$ such that every nonzero element of $A$ is of rank 1 , then $A$ is a minimal right ideal.

Proof. Let $a, b$ be two nonzero elements of $A$. Then $V=\operatorname{ker} a \oplus D u=$ ker $b \oplus D w$ for some nonzero vectors $u$ and $w$ since the ranks of $a$ and $b$ are one. If either $\operatorname{ker} a \subseteq \operatorname{ker} b$ or $\operatorname{ker} b \subseteq \operatorname{ker} a$ then $\operatorname{ker} a=\operatorname{ker} b$. Hence $u a \neq 0, u b \neq$ 0 and $u a r=u b$ for some $r \in R$. Thus $V(a r-b)=0$ and $a R=b R$ and, therefore, $A$ is a minimal right ideal. So suppose now that there exist $v_{1} \in(\operatorname{ker} a) \backslash(\operatorname{ker} b)$, $v_{2} \in(\operatorname{ker} b) \backslash(\operatorname{ker} a)$. There is $r \in R$ such that $v_{2}$ ar and $v_{1} b$ are linearly independent if the dimension of the space is greater than 1 . Since $a, b$ are elements of $A, a r+b \in A$. However, the rank of $a r+b$ is greater than or equal to 2 since $v_{1}(a r+b)=v_{1} b$ and $v_{2}(a r+b)=v_{2} a r$. This is a contradiction. 
2.2. Proposition. Let $R$ be a dense ring of linear transformations of a vector space $V$ over a division ring $D$ and let $A$ be a nonzero right ideal of $R$. Then $b(a)=b(A)$ for every nonzero $a \in A$ if and only if $A$ is a minimal right ideal.

Proof. Since $b(a)=b(a R)$ for every $a \in R$, if $A$ is a minimal right ideal, then certainly $b(a)=b(A)$. Assume now that $b(a)=b(A)$ for every nonzero $a \in A$. In order to prove that $A$ is minimal, it suffices to show that the rank of nonzero $a \in A$ is 1 . If the rank of $a$ is not 1 , then there exist vectors $v_{1}, v_{2}$ in $V$ such that $v_{1} a, v_{2} a$ are linearly independent. Hence there is $r \in R$ such that $v_{1} a r=0$ and $v_{2} a r \neq 0$. By hypothesis, $b(A)=b(a)=b(a r)$. Since $v_{1}^{\perp} \in X(R)$ and $v_{1}^{\perp} \epsilon$ $b(a r)=b(a), v_{1} a=0$. This is a contradiction.

2.3. Definition. If $R$ is a ring and $A$ is a right ideal of $R$, the radical of $A$ is $\sqrt{A}=\bigcap\{I \mid I \in X(R)$ and $I \supseteq A\}$.

2.4. Proposition, Let $R$ be a dense ring of linear transformations of a left vector space $V$ over a division ring $D$. If $A$ is a minimal right ideal of $R$, then $\sqrt{A}=A$.

Proof. Suppose $A \neq \sqrt{A}$. Since $A \subseteq \sqrt{A}$ always, if $A \neq \sqrt{A}$ then there is $b \in \sqrt{A}$ such that $b \notin A$. Since $R$ is semisimple, $X(R) \neq b(b)$ and $b(b) \supseteq b(\sqrt{A})$ $=b(A)$. Let $a$ be a nonzero element of $A$. Then $b(A)=b(a)$ since $A=a R$. Hence $b(b) \supseteq b(a)$. Let $\operatorname{ker} a=\{v \in V \mid v a=0\}$ and ker $b=\{v \in V \mid v b=0\}$. Then ker $a \subseteq$ ker $b$ since $b(b) \supseteq b(a)$. Since the rank of $a$ is $1, V=\operatorname{ker} a \oplus D w$ for some nonzero vector $w$ in $V$. Since $\operatorname{ker} a \subseteq \operatorname{ker} b$ and $b \neq 0, w b \neq 0$. Let $r \in R$ such that war $=w b$. Then $V(a r-b)=0$ and $a r=b$. Hence $b \in A$ and this is a contradiction.

2.5. Definition. Let $R$ be a ring. A closed subset $F$ of $X(R)$ is said to be a hyperplane of $X(R)$ provided that $j(F) \neq J(R)$, the Jacobson radical, and if $F^{\prime}$ is a closed subset of $X(R)$ such that $F^{\prime} \supseteq F$ and $F^{\prime} \neq F$ then $j\left(F^{\prime}\right)=J(R)$.

2.6. Theorem. Let $R$ be a primitive ring. If $A$ is a minimal right ideal of $R$, then $b(A)$ is a byperplane. If $F$ is a byperplance of $X(R)$ then $j(F)$ is a minimal right ideal of $R$.

Proof. Let $A$ be a minimal right ideal of $R$ and let $F=b(A)$. Let $F^{\prime}$ be a closed subset of $X(R)$ such that $F^{\prime} \supseteq F$ and $j\left(F^{\prime}\right) \neq 0$. Let $B=j\left(F^{\prime}\right)$. Then $0 \neq$ $B=j\left(F^{\prime}\right) \subseteq j(F)=\sqrt{A}=A$. By minimality of $A, B=A$, so $F^{\prime} \subseteq b(A)=F$ and $F=F^{\prime}$. Conversely, assume that $F$ is a hyperplane. Let $A=j(F)$. If $a$ is a nonzero element of $A$, then $j(b(a)) \neq 0$ and $b(a) \supseteq b(A) \supseteq F$. It follows that $b(a)$ $=b(A)=F$. Hence by $2.2, A$ is a minimal right ideal of $R$.

2.7. Corollary. Let $R$ be a primitive ring. Let $\Sigma_{1}$ be the set of minimal right ideals of $R$. Let $\mathcal{F}=\{F \mid F$ is a closed subset of $X(R)$ such that $j(F) \neq 0\}$. 
Let $\Sigma_{2}$ be the set of maximal elements of $\mathfrak{F}$. Then there is a bijection from $\Sigma_{1}$ onto $\Sigma_{2}$.

Proof. The mapping $A \rightarrow b(A)$ for $A \in \Sigma_{1}$ is a bijection from $\Sigma_{1}$ onto $\Sigma_{2}$.

2.8. Theorem. If $R$ is a primitive ring sucb that $X(R)$ is irreducible and $A$ is a minimal right ideal of $R$, then either $b(A)=\varnothing$ or $b(A)$ is a maximal proper irreducible closed subset of $X(R)$.

Proof. If $A$ is a minimal right ideal of a primitive ring $R$ then there is an idempotent $e \in R$ such that $A=e R$, and if $b(A) \neq \varnothing$ then $e^{l}=\{r \in R \mid r e=0\}$ is a nonzero left ideal of $R$. Hence $V_{0}=e^{l} \cap A \neq 0$ and $V_{0}$ is a subspace of the vector space $e R$ over the division ring $e R e$. Since $R$ is a dense ring of linear transformations of the space $e R$ and $X(R)$ is irreducible, by [3, 2.4] $e R e$ is an infinite field. Since $A$ is minimal, by $2.2 b(a)=b(A)$ if $a$ is a nonzero element of $A$. Let ker $a=\{x \in A \mid x a=0\}$. Then $V_{0}=\operatorname{ker} a$. Since the rank of $a$ is 1 , there is a vector $u$ such that $e R=V_{0} \oplus e R e u$. Hence $V_{0}^{\perp} \cap u^{\perp}=0$ and $V_{0}^{\perp}$ is a minimal right ideal of $R$ since $u^{\perp}$ is a maximal right ideal of $R$. Since $V_{0}^{\perp} \supseteq$ $a R$ and $V_{0}^{\perp}$ is a minimal right ideal, $V_{0}^{\perp}=a R$. Now suppose that $b(A)$ is reducible. Then by $[3,1.11]$ there exist right ideals $A_{1}, A_{2}, \ldots, A_{n}$ such that $b(A)$ $\not b\left(A_{i}\right)$ for every $i$ and $b(A)=\bigcup_{i=1}^{n} b\left(A_{i}\right)$. Let $W_{i}=\left\{v \in e R \mid v A_{i}=0\right\}$ for every $i$. Then $W_{i}$ is a subspace of $V_{0}$ and $V_{0}=\bigcup_{i=1}^{n} W_{i}$. If $V_{0}=W_{i}$ for some $i$, then $V_{0}^{\perp}=W_{i}^{\perp}=a R$, and $b(A)=b(a R)=b\left(W_{i}^{\perp}\right)$. Since $A_{i} \subseteq W_{i}^{\perp}, \stackrel{b}{b}\left(A_{i}\right) \supseteq b\left(W_{i}^{\perp}\right)=$ $b(A)$. This is a contradiction.

2.9. Lemma. Let $R$ be a simple ring with nonzero socle. If $X(R)$ is a discrete space then either $R$ is a division ring or $R$ is a finite ring.

Proof. Suppose that $R$ is neither a division ring nor a finite ring. By [3, 2.6], $R$ is isomorphic to a dense ring of linear transformations of finite rank of a vector space $V$ over a finite field $D$ and hence $1 \notin R$. Let $p$ be the characteristic of $D$ then $p r=0$ for every $r \in R$. Let $\mathrm{Z} /(p)$ be the field of integers modulo $p$. Then $R$ is an algebra over $\mathrm{Z} /(p)$. Let $R^{*}=\mathrm{Z} /(p) \times R$ be the usual extension ring of $R$ with 1 in which $R$ is an ideal. Let $m$ be a maximal regular right ideal of $R$ and let $e$ be a left identity modulo $m$ in $R$. Then $I(m, e)$ is a $\max$ imal right ideal of $R^{*}$ by 1.10. Let $S$ be an ideal of $R^{*}$ such that $S \subseteq I(m, e)$. If $S$ were a nonzero ideal then $S \cap R=0$ since $m$ contains no nonzero ideal of $R$. Hence $S R=0$. Let $\left(f, s_{0}\right)$ be a nonzero element of $S$ for some $f \in \mathbf{Z} /(p)$ and $s_{0} \in R$. Then $f \neq 0$ and $\left(f, s_{0}\right)(0, r)=(0,0)$ for every $r \in R$. Hence $-\left(f^{-1} s_{0}\right) r$ $=r$ for every $r \in R$. Since $R$ is a simple ring, $-\left(f^{-1} s_{0}\right)=1$ and $R$ must be a finite ring. Therefore, $S=0$ and $R^{*} / I(m, e)$ is a faithful simple $R^{*}$-module. Thus $R^{*}$ is a primitive ring with 1 . Observe $b(R)=\{R\}$ in $X\left(R^{*}\right)$ and 
$X\left(R^{*}\right)=\operatorname{supp}(R) \cup\{R\}$. Since $X(R)$ is homeomorphic to supp $(R)$ in $X\left(R^{*}\right)$ by 1.12 and $X(R)$ is a discrete space, every point of $\operatorname{supp}(R)$ is open in $X\left(R^{*}\right)$. Since the point $R$ in $X\left(R^{*}\right)$ can be separated from any point of $\operatorname{supp}(R)$ by open sets, this means that $X\left(R^{*}\right)$ is a Hausdorff space. Since $R^{*}$ is a primitive ring with 1 , by $[3,2.7] R^{*}$ must be a finite ring and therefore $R$ must be also a finite ring. This is a contradiction.

2.10. Theorem. Let $R$ be a primitive ring. Then $X(R)$ is a discrete space if and only if either $R$ is a division ring or a finite ring.

Proof. Since $X(R)$ is a $T_{1}$-space, if $R$ is a finite ring, then certainly $X(R)$ is a discrete space. Suppose $X(R)$ is a discrete space such that $R$ is not a division ring. Then $X(R)$ is a Hausdorff space and, by [3, 2.5], $R$ is a dense ring with nonzero socle of linear transformations of a vector space of dimension two or more over a finite field and if $S$ is the socle of $R$ then $R / S$ is a radical ring. If $S$ is a finite ring, then $R$ must be also a finite ring since $X(R)=\operatorname{supp}(S) \cup$ $b(S)=\operatorname{supp}(S)$ and since a primitive ring which is not a division ring with a $\mathrm{f}$ nite number of maximal regular right ideals is a finite ring. Now observe that $S$ satisfies the hypothesis of 2.9. Hence if $X(R)$ is a discrete space, then so is $X(S)$ and, by $2.9, S$ is a finite ring.

2.11. Example. Let $\mathrm{Z}$ be the ring of integers and let $R$ be the ring of all row-finite infinite matrices over $\mathbf{Z} /(2)$ of the following form:

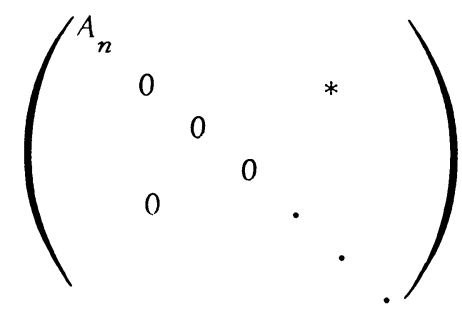

where $A_{n}$ is an $n \times n$ matrix for some positive integer $n$. Then $R$ is a primitive ring with nonzero socle and if $S$ is the socle of $R$ then $R / S$ is a radical ring. Hence $X(R)$ is a Hausdorff space by $[3,2.5]$ and it is not a discrete space by 2.10 .

3. Rings in which every proper right ideal is incompressible. Let $I, J$ be two distinct maximal regular right ideals of a ring $R$. If $K, M$ are distinct maximal regular right ideals of $R$ such that $\{K, M\} \subseteq b(I \cap J)$, then $(K \cap M) /(I \cap J)$ is a submodule of the right $R$-module $R /(I \cap J)$ which is isomorphic to a direct sum of two simple modules $I /(I \cap J)$ and $J /(I \cap J)$. Hence if $(K \cap M) /(I \cap J)$ is not the zero submodule then either $(K \cap M) /(I \cap J)=R /(I \cap J)$ or $(K \cap M) /(I \cap J)$ is isomorphic to $I /(I \cap J)$ or to $J /(I \cap J)$. In any case, this 
means that either $R /(K \cap M)$ is simple or $R /(K \cap M)=0$. This, of course, is absurd. Thus $K \cap M=I \cap J$ and $b(K \cap M)=b(I \cap J)$.

3.1. Definition. If $x, y$ are two distinct points in $X(R)$, a line containing $x$ and $y$ is $l(x, y)=b(j(x) \cap j(y))$.

3.2. Proposition. Given two distinct points in $X(R)$, there is one and only one line containing these two points.

Proof. Let $x, y$ be two distinct points in $X(R)$. If $z, w$ are distinct points in $X(R)$ such that $\{z, w\} \subseteq l(x, y)$, then $l(z, w)=l(x, y)$ since $j(z) \cap j(w)=j(x)$ $\cap j(y)$.

3.3. Proposition. If $x, y$ are two distinct points in $X(R)$ such that $R / j(x)$ $\cong R / j(y)$ as $R$-modules, then for every $w \in l(x, y), R / j(w) \cong R / j(x)$.

Proof. If $w \in l(x, y)$, then $j(w) /(j(x) \cap j(y))$ is a nonzero submodule of $R /(j(x) \cap j(y))$. Hence $j(w) /(j(x) \cap j(y))$ is either isomorphic to $R / j(x)$ or to $R / j(y)$.

3.4. Lemma. Let $R$ be a ring and $M$ be a simple $R$-module. Let $D=$ $\operatorname{Hom}_{R}(M, M)$. If $m_{1}, m_{2}$ are nonzero elements of $M$ sucb that $m_{1}^{\perp} \neq m_{2}^{\perp}$ then $b\left(j\left(m_{1}^{\perp}\right) \cap j\left(m_{2}^{\perp}\right)\right)$ contains at least three elements.

Proof. Since $M$ is a left vector space over a division ring $D$, if $m$ is a nonzero element of $D m_{1} \oplus D m_{2}$ then $m^{\perp} \in b\left(j\left(m_{1}^{\perp}\right) \cap j\left(m_{2}^{\perp}\right)\right)$, and if $m=m_{1}+m_{2}$ then $m^{\perp} \neq m_{1}^{\perp}$ and $m^{\perp} \neq m_{2}^{\perp}$.

3.5. Theorem. Let $R$ be a ring. Then there is exactly one isomorphic class of simple $R$-modules if and only if $X(R)$ bas no line which contains exactly two points.

Proof. Assume that $R$ has a unique isomorphic class of simple modules. If there is a two-point line, say $l(x, y)$, for some two distinct points $x$ and $y$ in $X(R)$, then $b(j(x) \cap j(y))=\{x, y\}$. Let $M=R / j(x)$. Then $x=m_{1}^{\perp}$ and $y=m_{2}^{\perp}$ for some $m_{1}, m_{2}$ in $M$ and $M^{\perp}=J(R)$ since all simple $R$-modules are isomorphic. By $3.4, l(x, y)$ contains at least 3 points. This is a contradiction. Conversely, suppose that $R / I_{1} \cong M_{1} \not M_{2} \cong R / I_{2}$ are simple modules where $I_{1}, I_{2} \in X(R)$. If there exists maximal regular right ideal $K$ such that $K \supseteq I_{1} \cap I_{2}$ and $I_{j} \neq K$ for $j=1,2$, then by the remark at the beginning of $\$ 3, R \Lambda_{1}=\left(K+I_{1}\right) / I_{1} \cong$ $K /\left(I_{1} \cap K\right)=K /\left(I_{2} \cap K\right) \cong\left(K+I_{2}\right) / I_{2}=R / I_{2}$, a contradiction. Thus the line containing $I_{1}$ and $I_{2}$ contains exactly two points.

3.6. Proposition. If $R$ is a ring with 1 and if there is exactly one isomorphic class of simple $R$-modules then $R / J(R)$ is a simple ring. There is a primitive 
ring with nonzero socle in which every pair of simple modules are isomorphic, but the ring is not simple.

Proof. If $R / J(R)$ is not a simple ring, then there is an ideal $S$ in $R$ such that $S \supseteq J(R)$ but $S \neq J(R)$ and $R \neq S$. Let $I$ be a maximal right ideal of $R$ such that $I \supseteq S$. Then $R / I$ is a simple $R$-module and $(R / I)^{\perp} \supseteq S$. Since every pair of simple $R$-modules are isomorphic, if $M$ is a simple $R$-module, then $M^{\perp} \supseteq S$. Hence $J(R) \supseteq S$. This is a contradiction. Now, let $R$ be the primitive ring in Example 2.11. If $S$ is the socle of $R$ then $R / S$ is a radical ring. Hence if $x \in X(R)$, $R / j(x)$ is isomorphic to a minimal right ideal of $R$. Thus every pair of simple $R$ modules are isomorphic. However, $R$ is not a simple ring.

3.7. Theorem. Let $R$ be a primitive ring. Then $R$ is simple artinian if and only if (i) $X(R)$ is compact, (ii) $X(R)$ bas no two-point line and (iii) $X(R)$ bas a byperplane.

Proof. Assume (i), (ii) and (iii). Then $R$ has nonzero socle by 2.6. Let $S$ be the socle of $R$. Since $X(R)$ has no two-point line, by 3.5 all simple $R$-modules are isomorphic. Hence every simple module is isomorphic to a minimal right ideal. Therefore, $R / S$ is a radical ring. Thus by $1.12, X(S)$ is compact and by $1.16,1 \in S$. Since the unity of the ring $S$ is also the unity of $R, R=S$ and $R$ is a simple artinian ring. Conversely, if $R$ is a simple artinian ring then $1 \in R$. Hence $X(R)$ is compact by $[3,1.7]$. Since every pair of $R$-modules in a simple artinian ring are isomorphic, by 3.5, (ii) is true. Finally (iii) is true by 2.6 .

3.8. Theorem. Let $R$ be a semisimple ring. Then the following two statements are equivalent:

(i) There exist at least two points in $X(R)$ and every line contains an inf $i$ nite number of points.

(ii) $R$ is a dense ring of linear transformations of a vector space of dimension two or more over an infinite division ring sucb that every pair of simple $R$ modules are isomorpbic.

Proof. Assume (i). Then by 3.5 there is exactly one isomorphic class of simple $R$-modules. Hence if $M_{1}, M_{2}$ are two simple $R$-modules then $M_{1}^{\perp}=M_{2}^{\perp}$. Thus $M_{1}^{\perp}=J(R)=0$. Therefore, every simple $R$-module is faithful and, in particular, $R$ is a primitive ring. Let $M$ be a simple $R$-module and let $D$ be the endomorphism ring of $R$-module $M$. Since $X(R)$ contains at least two points, the dimension of the vector space $M$ over $D$ is at least two. Let $m_{1}, m_{2}$ be two linearly independent vectors in $M$. Then $m_{1}^{\perp} \neq m_{2}^{\perp}$ and the line $l\left(m_{1}^{\perp}, m_{2}^{\perp}\right)$ contains an infinite number of points by hypothesis. If $x \in l\left(m_{1}^{\perp}, m_{2}^{\perp}\right)$ then $x=m^{\perp}$ for some $m \in M$ since $M \cong R /(j(x))$ and hence $m \in D m_{1} \oplus D m_{2}$ by [1, Lemma, p. 27]. 
Conversely, if $m$ is a nonzero element of $D m_{1} \oplus D m_{2}$, then $m^{\perp} \in l\left(m_{1}^{\perp}, m_{2}^{\perp}\right)$. Thus $D$ must be an infinite division ring. Assume, now, (ii). Let $R$ be a dense ring of linear transformations of a vector space $M$ over an infinite division ring $D$ of dimension two or more, then $X(R)$ contains at least two points. Let $x$ and $y$ be two distinct points in $X(R)$. Then $x=m_{1}^{\perp}$ and $y=m_{2}^{\perp}$ for some $m_{1}, m_{2}$ in $M$ since $M \cong R / j(x) \cong R / j(y)$. Hence the line $l(x, y)=b\left(m_{1}^{\perp} \cap m_{2}^{\perp}\right)$. Let $W$ be the two-dimensional subspace of $M$, which is spanned by $m_{1}$ and $m_{2}$. Observe that $z \in l(x, y)$ if and only if $z=w^{\perp}$ for some $0 \neq w \in W$. Since $D$ is infinite and $\left(m_{1}+\alpha m_{2}\right)^{\perp} \neq\left(m_{1}+\beta m_{2}\right)^{\perp}$ for $\alpha \neq \beta$ in $D, W$ contains infinitely many vectors. Thus $l(x, y)$ contains an infinite number of points.

3.9. Theorem. Let $R$ be an arbitrary ring. Then every line in $X(R)$ con. tains exactly two points if and only if every maximal regular right ideal is also a left ideal.

- Proof. Assume that every line in $X(R)$ contains exactly two points. Let $l$ be a maximal regular right ideal of $R$. Let $N(I)=\{r \in R \mid r I \subseteq I\}$. Then by [1, Theorem 1, p. 25], $N(I) / I=\operatorname{Hom}_{R}(R / I, R / I)$ and $R / I$ is a left vector space over the division ring $N(I) / I$. If the dimension of the vector space $R / I$ over $N(I) / I$ is greater than one, then by 3.4 there will be a line which contains more than two points. Hence $R / I$ is one dimensional. Let $e$ be a left identity modulo $I$. Then for every $a \in R$ there is $b \in N(I)$ such that $b e-a \in I$. Since $b e \in N(I), a \in N(I)$. Thus $I$ is a left ideal. Conversely, if every maximal regular right ideal of $R$ is also a left ideal, then $I J \subseteq I \cap J$ for every pair of maximal regular right ideals $I$ and $J$. Hence if $K \in b(I \cap J)$ then $I J \subseteq K$ and either $I=K$ or $J=K$ by 1.7. Thus $b(I \cap J)=\{I, J\}$.

3.10. Proposition. Let $R$ be an arbitrary ring. Assume that every proper right ideal of $R$ is an intersection of maximal regular right ideals. Then for every right ideal $A, A R=A$.

Proof. If $R^{2} \neq R$, then by hypothesis, $R^{2}$ must be an intersection of maximal regular right ideals. This is of course impossible in view of 1.7. Therefore, $R^{2}=R$. If $A$ is a proper right ideal of $R$ then $A R=\sqrt{A R}$ and $A=\sqrt{A}$. If there is a maximal regular right ideal $I$, such that $A R \subseteq I$, then by $1.7, A \subseteq I$ so $\sqrt{A R}$ $=\sqrt{A}$. Hence $A R=A$.

3.11. Definition. Let $R$ be an arbitrary ring. An $R$-module $M$ is called injective if and only if for every pair $A, B$ of $R$-modules with $A \subseteq B$, each homomorphism of $A$ into $M$ can be extended to one of $B$ into $M$. If $R$ is a ring with 1 and if $M$ is a unital $R$-module, then it is well known that $M$ has a minimal injective extension which is also a maximal essential extension of $M$. Such minimal injective extension is unique up to an isomorphism. It has been shown by 
R. E. Johnson $[2,7.1]$ that these results are also true for any ring $R$ and any $R$ module $M$. For each $R$-module $M$, we denote by $\hat{M}$ the minimal injective extension of $M$.

3.12. Proposition. Let $R$ be a ring. If every simple $R$-module is injective, then for any simple module $M$ the R-homorphism $\phi: M \rightarrow \operatorname{Hom}_{R}(R, M)$ where $\phi(m)(r)=m r$ for every $m \in M$ and $r \in R$ is an isomorphis $m$.

Proof. Clearly, $\phi$ is a monomorphism. Let $f \in \operatorname{Hom}_{R}(R, M)$. Let $R^{\#}$ be an extension ring of $R$ with 1 . Then there is $\bar{f} \in \operatorname{Hom}_{R}\left(R^{\sharp}, M\right)$ such that $\left.\bar{f}\right|_{R}=f$ since $M$ is injective. Let $\bar{f}(1)=m_{0}$. Then $f(r)=m_{0} r$ for every $r \in R$. Therefore, $f=\phi\left(m_{0}\right)$ and $\phi$ is an isomorphism.

3.13. Proposition. Let $R$ be a ring such that $R^{2}=R$. If every simple $R$ module is injective then every maximal right ideal of $R$ is regular.

Proof. Let $I$ be a maximal right ideal of $R$. Since $R^{2}=R, R / I$ is a simple $R$-module. Hence, by hypothesis, $R / I$ is injective and, by 3.12, $\phi(R / I)=$ $\operatorname{Hom}_{R}(R, R / I)$ where $\phi(r+I)\left(r^{\prime}\right)=r r^{\prime}+I$ for $r, r^{\prime} \in R$. Define $g: R \rightarrow R / I$ such that $g(r)=r+I$ for each $r \in R$. Then $g \in \operatorname{Hom}_{R}(R, R / I)$ and $g=\phi(a+I)$ for some $a \in R$. Thus $g(r)=a r+I$ for every $r \in R$ and hence $a r-r \in I$ for every $r \in R$.

3.14. Proposition. Let $R$ be a ring sucb that every maximal right ideal of $R$ is regular. If $M$ is a simple $R$-module such that $\hat{M} R=M$, then $\hat{M}=M$.

Proof. Consider $\phi: \hat{M} \rightarrow \operatorname{Hom}_{R}(R, \hat{M} R)$ as in 3.12. If the $\operatorname{ker} \phi$ were not zero then $\phi(M)=0$ and $M R=0$ since $\hat{M}$ is an essential extension of the simple module $M . \phi$ is a monomorphism. Let $f \in \operatorname{Hom}_{R}(R, \hat{M} R)$. Since $\hat{M} R \subseteq \hat{\hat{M}}$, there is $\bar{f} \in \operatorname{Hom}_{R}\left(R^{\#}, \hat{M}\right)$ such that $\bar{f}(r)=f(r)$ for every $r \in R$, where $R^{\#}$ is an extension ring of $R$ with 1 . Let $\bar{f}(1)=\hat{m}$ where $\hat{m} \in \hat{M}$. Then $f=\phi(\hat{m})$ and $\phi(\hat{M})$ $=\operatorname{Hom}_{R}(r, \hat{M} R)$. On the other hand, if $f \neq 0$ then $R / \operatorname{ker} f \cong \hat{M} R=M$. Hence ker $f$ is a maximal right ideal of $R$ and therefore, by hypothesis, it is regular. Let $e$ be a left identity modulo ker $f$. Then $f(e r)=f(r)=f(e) r$ for every $r \in R$ and $f=\phi(f(e))$. Hence $\phi(M)=\operatorname{Hom}_{R}(R, \hat{M} R)$. Thus $\phi(M)=\phi(\hat{M})$ and $M=\hat{M}$.

3.15. Remark. Villamayor [5] proved that if $R$ is a ring with 1 then every simple $R$-module is injective if and only if every proper right ideal of $R$ is the intersection of maximal right ideals.

3.16. Theorem. Let $R$ be an arbitrary ring. Then the following statements are equivalent:

(i) Every proper right ideal of $R$ is an intersection of maximal regular right ideals. 
(ii) If $A$ is a right ideal of $R$ then $A R=A$ and every simple $R$-module is injective.

Proof. Assume (i). Then by 3.10, $A R=A$ for every right ideal $A$ of $R$. Let $M$ be a simple $R$-module and let $0 \neq \hat{m} \in \hat{M}$. Then $(\hat{M})^{\perp} \neq R$. For if $(\hat{m})^{\perp}=R$ then the submodule $N=\{\hat{m} \in \hat{M} \mid \hat{m} R=0\}$ must contain $M$ since $\hat{M}$ is an essential extension of $M$ and $M$ is simple. Hence $M R=0$. This is, of course, impossible. Thus $(\hat{m})^{\perp} \neq R$ and $(\hat{m})^{\perp}=\bigcap_{a \in \mathbf{\Lambda}}\left\{I_{\alpha} \mid I_{\alpha}\right.$ is a maximal regular right ideal $\}$ for some index set $\Lambda$. Let $M_{\alpha}=R / I_{\alpha}$. Let $p_{\alpha}$ be the $\alpha_{\text {th }}$ projection of $\Pi_{\beta \in \mathbf{\Lambda}} M_{\beta} \rightarrow$ $M_{\alpha}$ and let $\mu: \hat{m} R \rightarrow \Pi_{\alpha \in \Lambda} M_{\alpha}$ be a map such that $p_{\alpha} \circ \mu(\hat{m} r)=r+I_{\alpha}$ for every $r \in R$. Then $\mu$ is a monomorphism. If there exists $a \in \Lambda$ such that $p_{a} \circ \mu$ is a monomorphism then $\hat{m} R$ is simple and $\hat{m} R=M$ since $M$ is the only simple submodule of $\hat{M}$. Now, if, for each $\alpha \in \Lambda, p_{a} \circ \mu$ is not a monomorphism, then $p_{\alpha} \circ \mu(M)=0$ for every $\alpha \in \Lambda$ and $\mu(M)=0$. Since $\mu$ is a monomorphis $m$, this means that $M=0$. This is impossible. Thus $\hat{M} R=M$ and, by $3.14, \hat{M}=M$ and (ii) holds. Now assume (ii). Let $A$ be a right ideal of $R$ such that $A \neq R$. Let $\left\{I_{\alpha} \mid \alpha \in \Lambda\right\}$ be the family of all maximal right ideals of $R$ which contain $A$. Suppose there exists $b \in \bigcap_{a \in \mathbf{\Lambda}} I_{a}$ such that $b \notin A$. Let $A_{0}$ be a right ideal of $R$ maximal with respect to the property that $A \subseteq A_{0}$ but $b \notin A_{0}$. Then the submodule of $R / A_{0}$ generated by $b+A_{0}$ is equal to $J / A_{0}$ for some right ideal $J$ which contains $A_{0}$ properly. Since $J R=J, J / A_{0}$ must be a simple $R$-module. Hence it is injective by hypothesis. Let $\phi$ be a homomorphism from $R / A_{0}$ onto $J / A_{0}$ such that $\left.\phi\right|_{J / A_{0}}=1_{J / A_{0}}$, the identity map on $J / A_{0}$. Such $\phi$ exists since $J / A_{0}$ is injective. Then $R / A_{0}=J / A_{0} \oplus \operatorname{ker} \phi$. If $\operatorname{ker} \phi \neq 0$ then $\phi\left(b+A_{0}\right)=0$. This is impossible. Thus $R / A_{0}=J / A_{0}$ and $A_{0}$ is a maximal right ideal of $R$. Hence $b \in A_{0}$. This is a contradiction. Thus, $A$ is an intersection of maximal right ideals. Since every maximal right ideal of $R$ is regular by 3.13, $A$ is an intersection of maximal regular right ideals.

3.17. Definition. A right ideal $C$ of a ring $R$ is said to be incompressible provided that if $\left\{A_{1}, A_{2}, \cdots, A_{n}\right\}$ is a finite set of right ideals such that $A_{i} \not$ $C$ for every $i$, then there is a maximal regular right ideal $I$ such that $C \subseteq I$ and $A_{i} \not \subset I$ for every $i$.

3.18. Remark. If $R$ is a commutative ring, then every prime ideal $P$ such that $R / P$ is semisimple is incompressible. For if $\left\{A_{1}, A_{2}, \cdots, A_{n}\right\}$ is a finite set of ideals such that $A_{i} \not \subset P$ for every $i$, then $A_{1} A_{2} \cdots A_{n} \not \subset P$. Hence there is a maximal ideal $I$ such that $P \subseteq I$ but $A_{1} A_{2} \cdots A_{n} \notin I$. Obviously in any ring every maximal regular right ideal is incompressible.

3.19. Proposition. If $C$ is an incompressible right ideal of a ring $R$, then $\sqrt{C}=C$. 
Proof. If $\sqrt{C} \neq C$, then there exists a maximal regular right ideal $I$ such that $C \subseteq I$ but $\sqrt{C} \not \subset I$. This is a contradiction.

3.20. Proposition. Let $R$ be a ring. Then a right ideal $C$ is incompressible if and only if $C=\sqrt{C}$ and $b(C)$ is an irreducible closed subset of $X(R)$.

Proof. Assume that $C$ is incompressible. Then $C=\sqrt{C}$ by 3.19. If $b(C)$ were reducible, then there exist right ideals $A_{1}, A_{2}, \cdots, A_{n}$ such that $b\left(A_{i}\right) \neq$ $b(C)$ and $b(C)=\bigcup_{i=1}^{n} b\left(A_{i}\right)$. Since $b\left(A_{i}\right) \subseteq b(C)$ and $b\left(A_{i}\right) \neq b(C), A_{i} \not \subset C$ for every $i$. Hence there is $I \in X(R)$ such that $C \subseteq I$ and $A_{i} \not \subset I$ for every $i$. This means that $b(C) \notin \bigcup_{i=1}^{n} b\left(A_{i}\right)$, which is a contradiction. Conversely, assume that $C=\sqrt{C}$ and $b(C)$ is irreducible. Let $\left\{A_{1}, A_{2}, \cdots, A_{n}\right\}$ be a finite set of right ideals such that $A_{i} \notin \subset$ for every $i$. If there is no maximal regular right ideal $I$ such that $C \subseteq I$ and $A_{i} \not \subset I$ for every $i$, then $b(C) \subseteq \bigcup_{i=1}^{n} b\left(A_{i}\right)$. Since $b(C)$ is irreducible, $b(C) \subseteq b\left(A_{i}\right)$ for some $i$. Hence $C \supseteq \sqrt{A_{i}} \supseteq A_{i}$. This is impossible.

3.21. Corollary. Let $R$ be a ring such that $R$ is not a radical ring. Then $X(R)$ is irreducible if and only if $J(R)$, the Jacobson radical, is incompressible.

3.22. Example. Let $R$ be a primitive ring with a minimal right ideal $A$. If $A=e R$ for some idempotent $e \neq 1$ in $R$ such that $e R e$ is an infinite division ring then $e R$ is incompressible. Because, in this case, $X(R)$ is irreducible by $[3,2.4]$ and $b(e R)$ is irreducible by 2.8 .

3.23. Theorem. Let $R$ be a ring. Then every proper right ideal of $R$ is incompressible if and only if (i) $A=A R$ for every right ideal $A$ of $R$, and (ii) either $R$ is a division ring or $R$ is a dense ring of linear transformations of a left vector space $V$ of dimension two or more over an infinite division ring $D$ such that $V$ is injective as an $R$-module and every simple $R$-module is isomorphic to $V$.

Proof. Assume that every proper right ideal of $R$ is incompressible. Then for every right ideal $A$ of $R, A=A R$ by 3.19 and 3.10. Suppose that $R$ is not a division ring. Let $x$ and $y$ be two distinct points in $X(R)$. Since $j(x) \cap j(y)$ is incompressible, $b(j(x) \cap j(y))$ is irreducible by 3.20. Hence $l(x, y)$ must contain an infinite number of points. Thus by 3.5, every pair of simple $R$-modules are isomorphic. Since zero ideal is incompressible, $R$ is semisimple and, since every pair of simple $R$-modules are isomorphic, every simple $R$-module is faithful. Hence $R$ must be a primitive ring and $X(R)$ is irreducible. Thus by $[3,2.4], R$ must be a dense ring of linear transformations of a vector space of dimension two or more over an infinite division ring. If every proper right ideal 
of $R$ is incompressible, then every proper right ideal of $R$ is an intersection of maximal regular right ideals by 3.19 . Thus by 3.16 , every simple $R$-module is injective. Conversely, assume (i) and (ii). Then by 3.16, if $A$ is a right ideal such that $A \neq R$ then $\sqrt{A}=\dot{A}$. Since every simple $R$-module is isomorphic to $V$, if $x \in X(R)$ then $x=v^{\perp}$ for some $0 \neq v \in V$. Hence there is subspace $W$ of $V$ such that $A=W^{\perp}$. If $b(A)$ were not irreducible then there exist right ideals $A_{1}, A_{2}, \cdots, A_{n}$ such that $b(A)=\bigcup_{i=1}^{n} b\left(A_{i}\right)$ and $b(A) \neq b\left(A_{i}\right)$ for every $i$. Let $W_{i}=\left\{w \in W \mid w A_{i}=0\right\}$ for each $i$. Then $W_{i}$ is a subspace of $W$ and $W=\bigcup_{i=1}^{n} W_{i}$. Note that $W_{i}$ is a proper subspace of $W$ since $b\left(A_{i}\right) \neq b(A)$. Hence by $[3,2.1]$ the division ring $D$ must be a finite field. This is impossible. Thus $A$ is incompressible by 3.20 .

Acknowledgment. The authors wish to thank the referee for many helpful suggestions.

\section{REFERENCES}

1. N. Jacobson, Structure of rings, 2nd rev. ed., Amer. Math. Soc. Colloq. Publ., vol. 37, Amer. Math. Soc., Providence, R. I., 1964. MR 36 \#5158.

2. R. E. Johnson, Structure theory of faithful rings. II. Restricted rings, Trans. Amer. Math. Soc. 84 (1957), 523-544. MR 18, 869.

3. K. Koh and J. Luh, Maximal regular right ideal space of a primitive ring, Trans. Amer. Math. Soc. 170 (1972), 269-277.

4. I. G. MacDonald, Algebraic geometry. Introduction to schemes, Benjamin, New York, 1968. MR 39 \#205.

5. G. O. Michler and O. E. Villamayor, On rings whose simple modules are injective (to appear).

6. J. von Neumann, On regular rings, Proc. Nat. Acad. Sci. U. S. A. 22 (1936), 707-713.

DEPARTMENT OF MATHEMATICS, NORTH CAROLINA STATE UNIVERSITY, RALEIGH, NORTH CAROLINA 27607 